\title{
Diagnosis of Keratitis by Cultivation, Fluorescence Microscopy and PCR has Uncovered that Acanthamoeba Emits Auto Fluorescence
}

\author{
Hadi Abd ${ }^{1,4}$, Amir Saeed ${ }^{1,2,3}$, Samir A Alharbi ${ }^{4}$ and Fawaz D Alshammari ${ }^{*}$ \\ ${ }^{1}$ University of Hail, college of Applied Medical Sciences, Department of Clinical Laboratory Sciences, Hail, Kingdom \\ of Saudi Arabia \\ ${ }^{2}$ Karolinska Institute, Department of Laboratory Medicine, Division of Clinical Microbiology, Stockholm, Sweden \\ ${ }^{3}$ University of Medical Sciences and Technology, Faculty of Medical Laboratory Sciences, Department of \\ Microbiology, Khartoum 11111, Sudan
}

${ }^{4}$ Al- Quweayiyah, Shaqra University, College of Applied Medical Sciences, Department of Medical Laboratory Sciences, Kingdom of Saudi Arabia

Received: 23 June, 2017; Accepted: 08 August, 2017; Published: 14 August, 2017

*Corresponding author: Fawaz D Alshammari, University of Hail, College of Applied Medical Sciences, Department of Clinical Laboratory Science, Hail, Kingdom of Saudi Arabia, Tel: +966 50545 0842; E-mail: fawazabof@gmail.com

\begin{abstract}
The amphizoic protozoa Acanthamoeba species are able to be free-living amoebae in environment or as parasites in humans and animals. These species comprise nearly 30 species and they have an increased role as human pathogens causing encephalitis in the nervous system or keratitis in the eyes. In diagnosis of a clinical sample of eye contact lens, light microscopy showed a heavy growth of amoeboid and Acanthamoeba trophozoites that occurred in agar plates and in cultivation flasks of patient sample and A. castellanii control as well. Fluorescence microscopy uncovered that visualised amoebae from patient and control samples emit fluorescence before- and after glutaraldehyde fixation. Polymerase Chain Reaction (PCR) and Gel electrophoresis identified that the isolated amoebic microorganisms were Acanthamoeba species. In conclusion, we have diagnosed a case of keratitis that caused by Acanthamoeba species by cultivating the eye contact lens sample using glutaraldehyde as fluorescent dye for fluorescence microscopy and PCR. Acanthamoeba trophozoites and cysts emit autofluorescence and glutaraldehyde can be used as fluorescent dye to enhance the autofluorescence of Acanthamoeba trophozoites and cysts for diagnosis purposes.
\end{abstract}

Keywords: Eye contact lens; Acanthamoeba keratitis; Fluorescence microscopy; Glutaraldehyde as fluorescent dye; Polymerase Chain Reaction; Autofluorescence

\section{Introduction}

Free-Living Amoebae (FLA) are environmental eukaryotes found worldwide in soil, air, and fresh or salt water. FLA comprise several genera such as Acanthamoeba, Balamuthia, Naegleria and Sappinia. The life cycle of Acanthamoeba has a reproductive trophozoite and a dormant cyst. The trophozoite has an oval to elongated shape with a diameter varies between 28 and $40 \mu \mathrm{m}$. The cyst has polygonal or star shape and measures between 15 to $28 \mu \mathrm{m}$ in diameter. Naegleria species have an additional flagellate stage [1].

Acanthamoeba, Balamuthia, Naegleria and Sappinia are human pathogens causing infections in the central nervous system (CNS), eyes, lungs and skin $[3,7,13,39]$. Naegleria fowleri causes Primary Amoebic Meningoencephalitis (PAM), which is a fulminant, necrotizing and haemorrhagic meningoencephalitis and later leading to death [26,39].
PAM is an acute infection and is generally fatal. Swimming in contaminated water exposes nasal mucosa to the organism, which can enter the CNS via the olfactory neuroepithelium and the cribriform plate. Most patients are healthy children or young adults [42].

Acanthamoeba and Balamuthia cause infections of the lungs and skin and Granulomatous Amoebic Encephalitis (GAE) [26]. In contrast to PAM, GAE is a more subacute or chronic infection in immunocompromised or debilitated individuals of all ages $[3,7,13,39,42]$. The entry portal is thoughtfully to be the skin or lower respiratory tract, with subsequent haematogenous dissemination to the CNS [37].

Acanthamoeba Keratitis (AK) is the vision-threatening corneal disease that it was recognized at first time in 1973 in the United States [28]. It is reported with an increased prevalence in different regions and countries year after year $[9,16,23,24,34,35,38,44]$. AK is usually an acute and progressive infection that becomes increasingly significant for human health worldwide [8]. It is documented that keratitis has been led to blindness in $15 \%$ of untreated cases [9]. The main risk factors that associated with $\mathrm{AK}$ are minor eye trauma and Contact Lens Wearing (CLW). Initially, patients suffer from severe ocular pain, photophobia, and a unilateral red eye [33].

Keratitis due to Acanthamoeba species is most commonly associated with poor contact lens hygiene [32]. However, there have been reports showed that keratitis can be caused by Acanthamoeba species in the tropics in non-contact lens wearers [14,36]. Blindness of the affected eye is frequently related to significant diagnostic delay because patients are treated initially as viral, bacterial, or fungal keratitis [17].

Glutaraldehyde is a three carbon molecule terminated at both ends by aldehyde groups (HCO) covalent bonds with amine groups of any adjacent protein to build the cross-linking. The glutaraldehyde is an extremely efficient fixative to preserve cellular structure and the fixation by crosslinking is a method commonly used for light and electron microscopy [19].

However, the aldehyde groups of glutaraldehyde contain double bonds between carbon and oxygen atoms $(\mathrm{O}=\mathrm{C})$. Electrons of the double bonds will be excited when exposed to light during the fluorescence microscopy and returned very quickly to lower energy. This process results in emitting 
fluorescence, which is always occurred in the same frequency, and therefore, this is why it emits autofluorescence when it used as a fixative for fluorescent labeled microorganisms under fluorescence microscopy [40].

Doctoral thesis of the first author of this article uncovers that trophozoites as well as cysts of $A$. castellanii emit a detectable autofluorescence [1]. This autofluorescence can be enhanced by glutaraldehyde fixation for Acanthamoeba detection purposes by fluorescence microscopy.

For diagnosis of Acanthamoeba in clinical sample of eye contact lens we used cultivation, glutaraldehyde as both fixative and fluorescent dye to visualize free living amoebae and Polymerase Chain Reaction (PCR) for identification of the isolated protozoa.

\section{Materials and Methods}

\section{Material}

An infected eye contact lens in $2 \mathrm{ml}$ sterile Phosphate Buffered Solution (PBS) sample from middle aged male contact lens wearer that was sent to Karolinska Institute, Department of Laboratory Medicine, Division of Clinical Microbiology, Stockholm, Sweden. As control, Acanthamoeba castellanii (ATCC 30234) was obtained from the American Type Culture Collection, Manassas, Virginia, USA.

\section{Cultivation of Patient Sample and Acanthamoeba Castellanii Positive Control}

Each of eye contact lens, pellet of the PBS solution and control sample was cultured separately on a non-nutrient agar plate seeded with Escherichia coli. The plates were sealed, incubated at $30^{\circ} \mathrm{C}$ and investigated daily by inverted microscope until amoebic growth occurred. Subsequently, a small piece of the agar from each plate was transferred to a relevant culture flask that contains $10 \mathrm{ml}$ of proteose peptone, yeast, glucose and minerals, growth medium no. 712 (American Type Culture Collection) supplemented with $100 \mathrm{IU} / \mathrm{ml}$ penicillin and $100 \mu \mathrm{g} / \mathrm{ml}$ streptomycin and incubated at $30^{\circ} \mathrm{C}$. When the concentration of amoebae reaches $10^{6}$ cells $/ \mathrm{ml}$, samples were taken to analysis by light microscopy, fluorescence microscopy and PCR.

\section{Light-and Fluorescence Microscopy}

One milliliter cell suspensions from sample flask and $A$. castellanii positive control were centrifuged for $10 \mathrm{~min}$ at $300 \mathrm{x}$ g. The pellets were suspended by $0.5 \mathrm{ml}$ PBS and prepared to light and fluorescence microscopy for the demonstration of FLA cells.

One milliliter cell suspensions from sample flask and $A$. castellanii positive control were centrifuged for $10 \mathrm{~min}$ at $300 \mathrm{x}$ g. The pellets were fixed with one milliliter $2.5 \%$ glutaraldehyde and examined by the fluorescence microscopy for the demonstration of fluorescent protozoa cells.

\section{DNA Extraction, DNA Amplification and Gel Electrophoresis}

Two milliliters cell suspensions from sample flask and $A$. castellanii positive control were diluted in $8 \mathrm{ml}$ of PBS, and centrifuged for $10 \mathrm{~min}$ at $300 \mathrm{~g}$. The pellet were suspended in $2 \mathrm{ml}$ of PBS solution, DNA extracted using the Qiagen DNA mini kit (Qiagen, Hilden,Germany).

DNA was amplified utilizing two primers sets that were forward primer 5'- GGC CCA GAT CGT TTA CCG TGA A-3' and reverse primer 5'TCT CAC AAG CTG CTA GGG GAGTCA-3'as previously used by Pasricha, et al. [30]. The amplification reaction was carried out in a final volume of $20 \mu \mathrm{l}$ containing each primer at a concentration of $0.3 \mu \mathrm{M}, 1.0 \times$ PCR golden buffer, $200 \mu \mathrm{M}$ deoxyribonucleoside triphospate, $1.2 \mathrm{mM} \mathrm{Mgcl}$, $1.25 \mathrm{U} / 50 \mu \mathrm{l}$ of Ampli Taq Gold (Sigma). The reaction was completed after 32 cycles of $95^{\circ} \mathrm{C}$ denaturation for $4 \mathrm{~min}, 55^{\circ} \mathrm{C}$ annealing for $20 \mathrm{sec}$, and $72^{\circ} \mathrm{C}$ for $10 \mathrm{sec}$ extension. Final reaction products were analyzed by electrophoresis on agarose gel in $1 \times$ TBE buffer (Tri base, boric acid and EDTA (pH 8.0). The gel was stained in $0.1 \%$ SYBR Green bath, visualized by UV transillumination, and photographed using Polaroid films. DNA fragment $487 \mathrm{bp}$ for Acanthamoeba was obtained as a final step.

\section{Results}

Different methods were performed to analyze the clinical sample in order to identify the causative agent of keratitis. These methods included amoeba culture, light and fluorescence microscopy and molecular methods.

\section{Heavy Growth of Amoeboid Cells on Agar Plates and in Cultivation Flasks}

Inverted microscopy showed heavy growth of amoeboid and Acanthamoeba trophozoites, which are occurred in both non-nutrient agar seeded with E. coli plates and in ATCC medium cultivation flasks of patient sample and in A. castellanii control, respectively.

\section{Visualization of Unfixed Amoeboid Cells by Light- and Fluorescence Microscopy}

Light microscopy disclosed that the growing trophozoites are characterized by amoeboid shapes with abundant cytoplasm (an ectocytoplasm and an endocytoplasm), which have pseudopodia. The cell has an obvious nucleus, large and small vacuoles in both patient sample

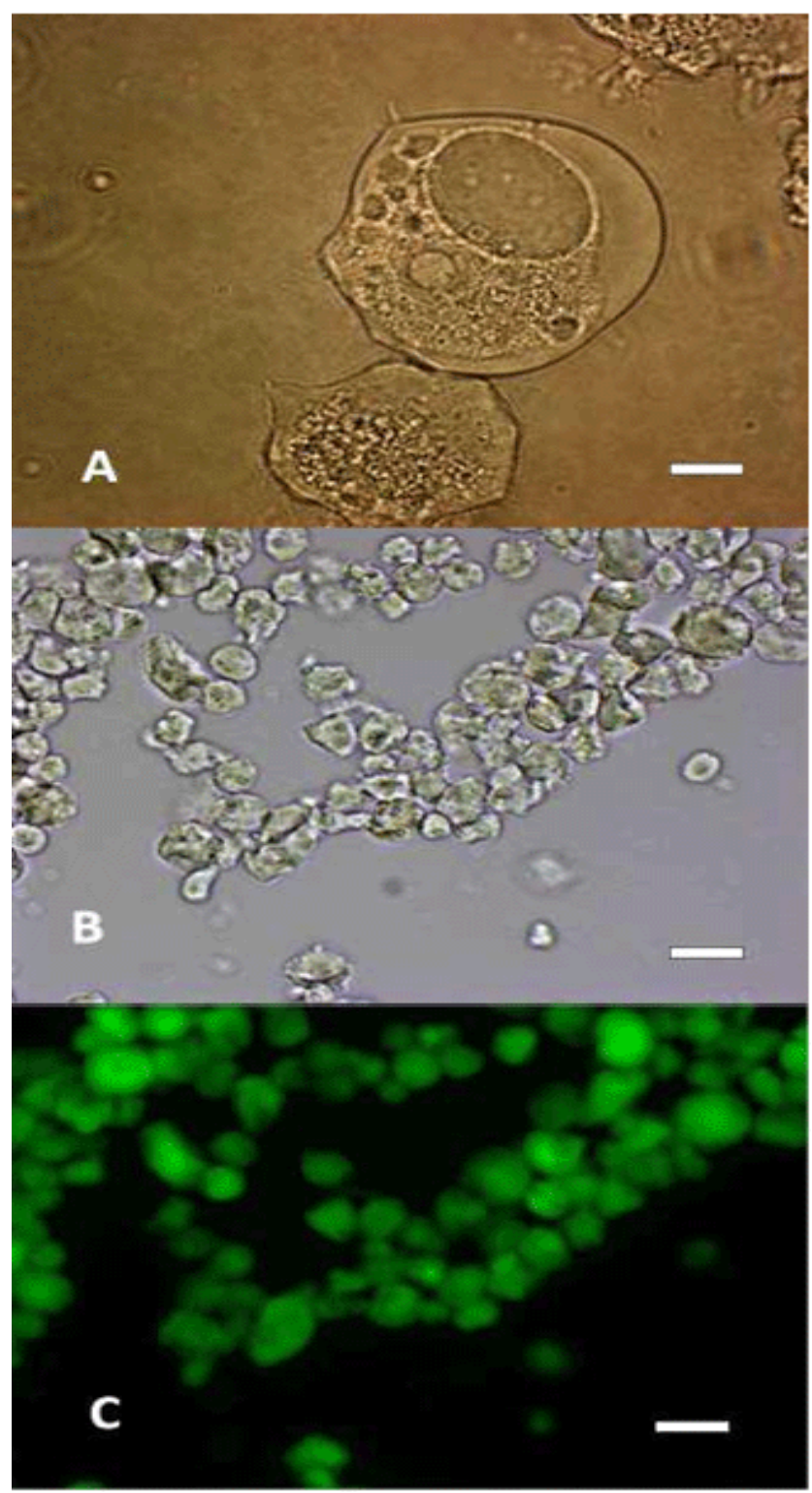

Figure 1: Light microscopy micrograph of growing amoeboid trophozoites of cultured patient sample. Bar is $10 \mu \mathrm{m}$ (A). Same wet preparation from patient cultured sample was visualized by light microscopy (B) and fluorescence microscopy (C). The fluorescence microscopy uncovered that the trophozoites emitted green autofluorescence. Bars are $25 \mu \mathrm{m}$ 
One milliliter cell suspension from sample flask was centrifuged as it was described in method section. The unfixed pellet prepared on a slide and examined by light- and fluorescence microscopy. Micrograph of protozoa cells visualised by the light microscopy was shown in (Figure 1B). The same slide was examined by the fluorescence microscopy and uncovered that the same protozoa cells emitted autofluorescence as it was shown in (Figure 1C).

\section{Visualization of Glutaraldehyde Fixed Amoeboid Cells by Fluorescence Microscopy}

One milliliter cell suspension from patient sample flask was centrifuged as it was described in method section. The pellets were fixed with one milliliter $2.5 \%$ glutaraldehyde and examined by the fluorescence microscopy for the demonstration of fluorescent amoeboid cells. The fixed cells from the patient samples showed fluorescent trophozoites and cyst that has characterised by double wall (ectocyst and endocyst) having a round form in comparison to unfixed cyst of $A$. castellanii emitting autofluorescence (Figure 2A,2B and 2C).

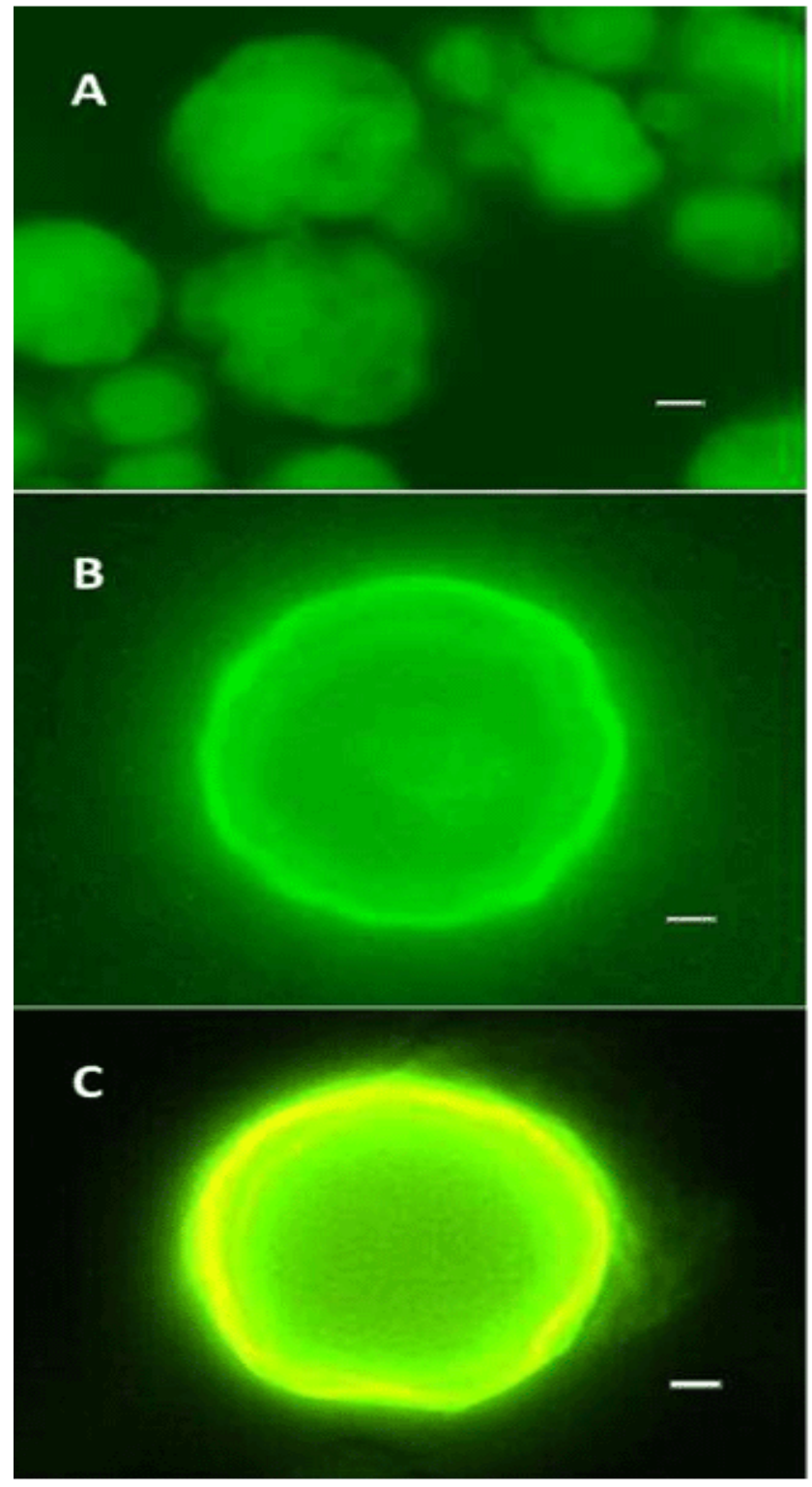

Figure 2: Fluorescence microscopy micrographs of Acanthamoeba: A. Trophozoites of $2.5 \%$ glutaraldehyde fixed preparation from cultured patient sample. Bar is $10 \mu \mathrm{m}$. B. Cyst of $2.5 \%$ glutaraldehyde fixed preparation from patient sample culture. Bar is $6 \mu \mathrm{m}$. C. Unfixed A. castellanii cyst emitting autofluorescence [1]. Bar is $6 \mu \mathrm{m}$

\section{Emission of Different Fluorescent Colors by the Growing Amoeboid Cells}

The growing amoeboid cells emitted 3 different fluorescent colors when blue, green, and triple filters of the fluorescence microscope utilized. Excitation wavelength rang of these fluorescence colors is from 380-558 nm. Each of blue filter, green filter, and triple filter visualized green, red and blue fluorescence, respectively (Figure 3A,3B and 3C).
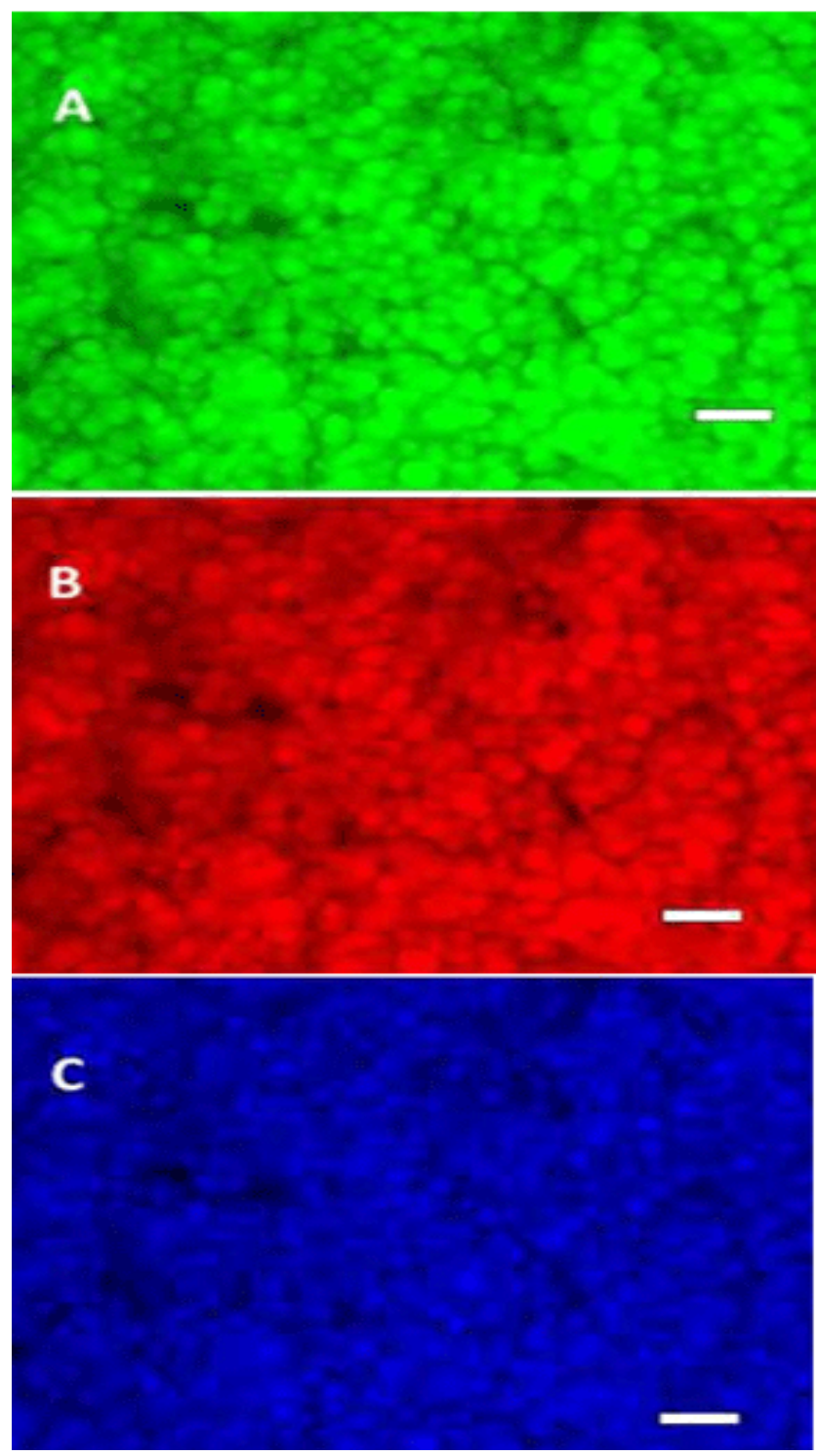

Figure 3: Acanthamoeba cells emit green, red and blue fluorescent colors according utilizing blue-, green-, and triple filter, respectively. Bars are $50 \mu \mathrm{m}$

\section{Visualization of Polymerase Chain Reaction Products by Gel Electrophoresis}

Molecular biology analysis included DNA extraction from $2 \mathrm{ml}$ cell culture of patient sample and $A$. castellanii positive control, amplification, visualization and identification of the isolated amoebic microorganisms as Acanthamoeba genus (Figure 4).

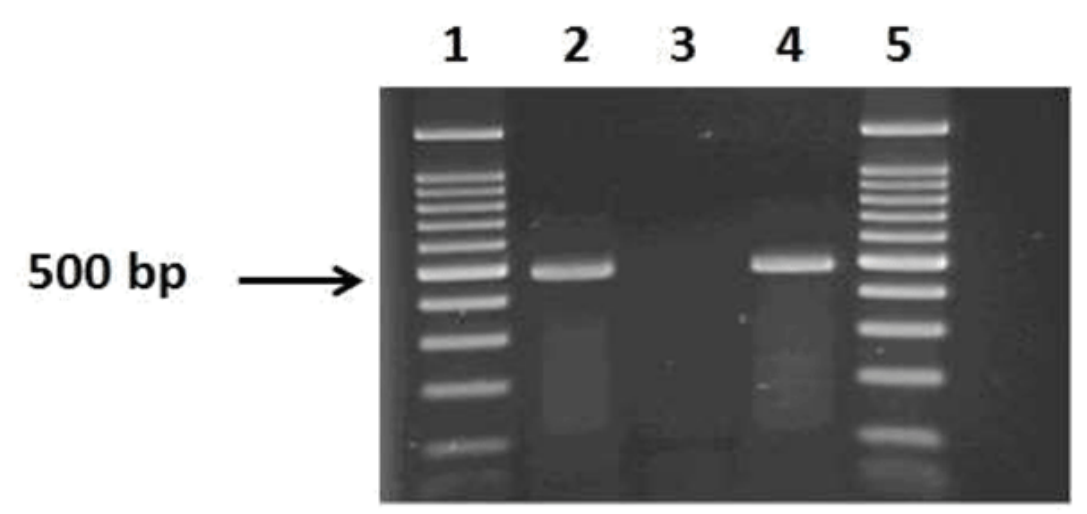

Figure 4: Agarose gel electrophoresis of PCR products of Acanthamoeba 18S rDNA gene. Lanes 1 and 5 are molecular mass marker (1500 bp), lane 2 amoebic positive control (approximately $450 \mathrm{bp}$ ), lane 3 amoebic negative control and lane 4 amoebic samples 


\section{Discussion}

Acanthamoeba keratitis (AK) was reported in 1973 in the United States [28]. The keratitis is a disease in which amoebae invade the cornea of the eye and its causative agent Acanthamoeba species became well recognized as human pathogen that can lead to blindness if it is not treated [9]. The reported first case of blindness due to Acanthamoeba was from Sudan and increasing of AK is observed in different regions and countries year after year $[9,17,16,23,24,34,35,38,44]$.

Eye contact lens wearers typically seek medical help late, because they are used to minor irritations in the eye [22]. In cases of severe infection, amoeba density is sometimes very high and the amoebae can already be detected by direct microscopy of the clinical samples without enrichment but by utilizing several staining methods such as methylene blue and Giemsa that enhance cellular intensity and differentiate between trophozoites and cysts of protozoa [22,38].

However, molecular biology methods such as mitochondrial DNA Restriction Fragment Length Polymorphism and zymodeme pattern analysis (isoenzyme electrophoresis) used in laboratory diagnosis of Acanthamoeba infection but these were not always reproducible methods $[10,11,45]$.

The direct detection of the causative agent in a corneal scrape specimen is only the reliable diagnostic method for AK and the cultivation method remains the gold standard of Acanthamoeba laboratory diagnosis [22]. However, if patients have already been pre-treated with antibiotics, the amoeba density is usually very low. Moreover, amoebae exhibit altered morphologies in these cases, even culture often remains negative and molecular techniques are indispensable. Reliable identification below the genus level requires genotyping [22]. It is claimed that the most accepted technique for the diagnosis of $\mathrm{AK}$, a part from the analysis of biopsies, is the use of in vivo confocal microscopy. It is a non-invasive tool with high sensitivity in cases of severe infection. But diagnosis confirmation requires other laboratory tests, mainly the cultivation of Acanthamoeba from corneal biopsy or the lens cases or contact lenses from the patient, as well as PCR or immunofluorescence assays [23].

In diagnosis of AK, Wilhelmus, et al. 1986 used Calco Flour White (CFW) staining and demonstrated amoebic cysts in corneal scrapings and keratectomy specimens from four patients with culture-proved Acanthamoeba keratitis and from one in which CFW was the only positive laboratory test. However, the CFW is a chemofluorescent dye with an affinity for the polysaccharide polymers of amoebic cysts [46].

It is known that some unicellular organisms such as amoebae, free living amoebae and giardia species are able to encyst as a protective response to a harmful environment. The cyst wall usually contains chitin as its main structural constituent. The chitin is the carbohydrate polymer conveying the required structural toughness to the cyst wall. But microorganisms of Acanthamoeba genus are exceptions, as their endocysts are made of cellulose compared to the ecto cyst that composed of proteins, lipids, and putative carbohydrate components as lectin binding sites $[5,6,29,41]$.The cellulose is a linear polymer of anhydrous glucose units. The hydroxyl groups of glucose units could be the coupling sites of the fluorescence dyes [47].

However, both polymers of chitin and cellulose form very similar crystalline macroscopic structures [4]. Specific cytochemical differentiation between cellulose and chitin by microscopy has not been possible due to the similarity of the constituent $\beta-1,4$-linked hexose backbones [21,12].

Chitin is an essential component of the cell walls and septa of all pathogenic fungi, and occurs in the cyst walls of pathogenic amoebae, the egg-shells and gut lining of parasitic nematodes and the exoskeletons of invertebrate vectors of human disease including mosquitoes, sand flies, ticks and snails [20]. While CFW is a special fluorescent stain that binds strongly to structures containing cellulose and chitin and fungiflora Y stain binds both fungi and acanthamoebae $[15,25,27,43,18]$ thus, the CFW and fungiflora Y stain are not specific dye for either Acanthamoebae or fungi.

Our current study utilized different methods such as amoeba cultivation, fluorescence microscopy and molecular methods to identify the causative agent of keratitis. These methods found that the causative agent was an amoeba belonged to Acanthamoeba genus and the identified Acanthamoeba found to emit autofluorescence. This finding was consistent with previous study by Abd, 2006 who found that fluorescence microscopy and flow cytometry analysis of $A$. castellanii cells population by FACSort, Becton Dickinson Immuno Systems, San Jose, CA, uncoverd that unlabeled $A$. castellanii cells adjusted for forward scatter (relative size) and side scatter FL1 (green colour) emitted detectable fluorescence, as in (Figure 2C and Figure 5)[1].

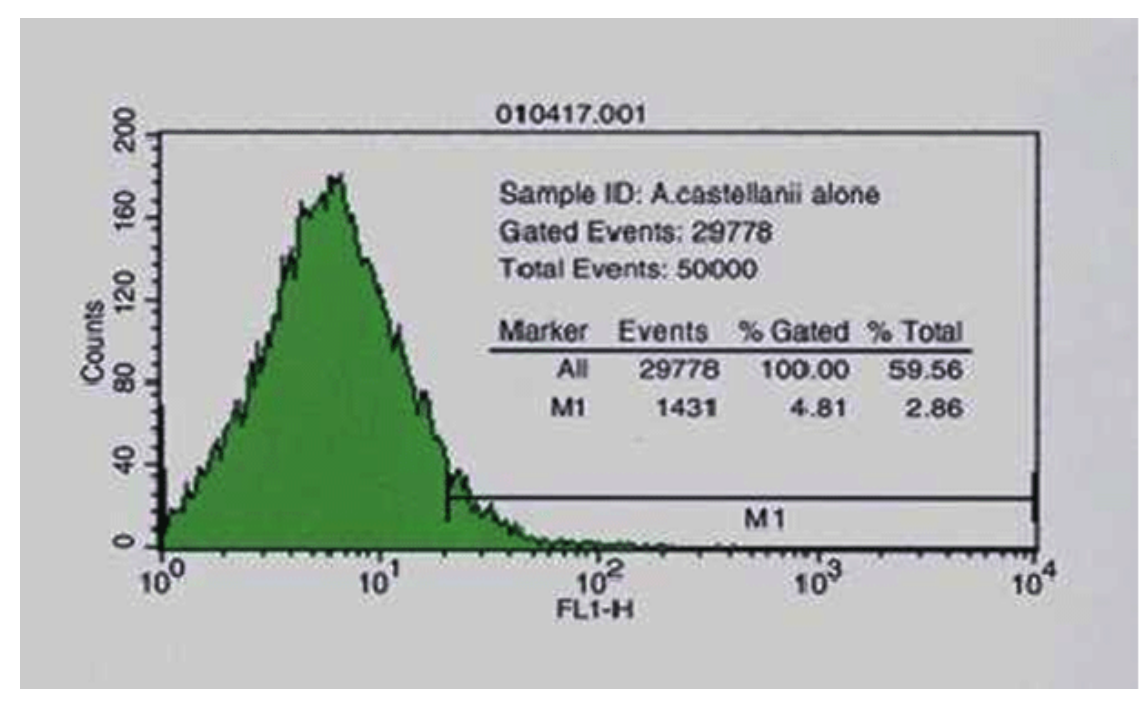

Figure 5: FACS analysis showing autofluorescence from A. castellanii cells population at day 15. The flow cytometer (FACSort, Becton Dickinson Immunosystems, San Jose, CA), equipped with an argon laser giving a 488-nm primary emission line, was calibrated using unlabeled and labeled beads (Becton Dickinson) and FACSComp software (Becton Dickinson) [1]

Also our finding of the autofluorescence emitted by Acanthamoeba cells was consistent with findings of other researcher who found that chitin, collagen and elastin in animal cells or cellulose in plants and fungi emit autofluorescence when excited by ultraviolet, violet, or blue light under 400-520 nm utilizing commercial fluorescent dyes [2,31].

However, the commercial diagnostic fluorescent dyes such as calcoflour white stain and fungiflora Y stain include fluorescence molecules added to their structures and these molecules generate the fluorescence to differ from glutaraldehyde $[15,18,25,27,43]$. The glutaraldehyde molecule structure has electrons of the double bonds that will be excited when exposed to light and emit autofluorescence when returned very quickly to lower energy. Therefore glutaraldehyde is a fluorescent dye and a fixative too according to its chemical structure $[40,19]$.

\section{Conclusions}

Utility of Acanthamoeba autofluorescence and glutaraldehyde enhanced fluorescence as a new method of diagnosis of Acanthamoeba trophozoites and cysts together with cultivation and molecular biology methods.

Emitting of green, red and blue autofluorescence uncovers new biological characteristics of Acanthamoeba.

Glutaraldehyde can be used as fixative and fluorescent dye for better visualization of protozoa and fungi. 


\section{References}

1. Abd H. Interaction between water-borne pathogenic bacteria and Acanthamoeba castellanii. Doctoral thesis. 2006.

2. Andersson H, Baechi T, Hoechl M, Richter C. Autofluorescence of living cells. J Microsc. 1998;191:1-7.

3. Bakardjiev A, Azimi PH, Ashouri N, Ascher DP, Janner D, Schuster FL. Amoebic encephalitis caused by Balamuthia mandrillaris: report of 4 cases. Pediatr Infect Dis J. 2003;22(5):447-453.

4. Blackwell J. Physical methods for the determination of chitin structure and conformation. Methods Enzymol. 1988;161:435-442.

5. Blanton WE, Villemez CL. Molecular size and chain length distribution in Acanthamoeba cellulose. J. Protozol. 1978;25(2):264-267. doi: 10.1111/ j.1550-7408.1978.tb04412.x

6. Bose K, Ghosh DK, Bhattacharya A. Membrane carbohydrate characterization of Acanthamoeba astronyxis, A. castellanii and Naegleria fowleri by fluoresceinconjugated lectins. Int J Parasitol. 1989;19(7):737-741.

7. Cermeno JR, Hernandez I, El Yasin H, Tinedo R, Sanchez R, Perez G, et al Meningoencephalitis by Naegleria fowleri. epidemiological study in Anzoategui state, Venezuela. Rev Soc Bras Med Trop. 2006;39(3):264-268.

8. Chomicz L, Szaflik JP, Padzik M, Izdebska J. Acanthamoeba Keratitis: The Emerging Vision-Threatening Corneal Disease. 2016. doi: 10.5772/64848

9. Clarehout I, Keystyln PH. Acanthamoeba keratitis: a review. Bull Soc Belgium Clin Microbiol Rev.2003;2:273-307.

10.Costas M, Griffiths AJ. The esterase and acid phosphatases of Acanthamoeba Protisologca. 1984;20:33-41.

11.De Jonckheere JF. Isoenzyme and total protein analysis by agarose isoelectric focusing and taxonomy of Acanthamoeba. J Protozol. 1983;30:701-706. doi 10.1111/j.1550-7408.1983.tb05346.x

12.Derda M, Winiecka-Krusnell J, Linder MB, Linder E. Labeled Trichoderma reesei cellulase as a marker for Acanthamoeba cyst wall cellulose in infected tissues. Appl Environ Microbiol. 2009;75(21):6827-6830.

13.Gelman BB, Rauf SJ, Nader R, Popov V, Borkowski J, Chaljub G. Amoebic encephalitis due to Sappinia diploidea. JAMA. 2001;285(19):2450-2451.

14.Hansen B, Kronborg G. Acanthamoeba keratitis in a non-contact lens wearer with human immunodeficiency virus. Scand J Infect Dis. 2003;35(3):207-209.

15.Herth W, Schnepf E. The fluorochrome, calcofluor white, binds oriented to tructural polysaccharide fibrils. Protoplasma. 1980;105(1-2):129-133.

16.Ibrahim YW, Boase DL, Cree IA. Factors affecting the epidemiology of Acanthamoeba keratitis. Ophthalmic Epidemiol. 2007;4:53-60. doi $10.1080 / 09286580600920281$

17.Imam AM, Mahgoub el S. Blindness due to Acanthamoeba: first case report from Sudan-Case Report. Int J Health Sci. 2008;2:163-166.

18.Inoue T, Asari S, Tahara K, Kiritoshi A, Inoue Y, Shimomura Y. Utility of Fungiflora $\mathrm{Y}$ stain in rapid diagnosis of Acanthamoeba keratitis. Br J Ophthalmol 1999;83:628-633.

19.Kiernan JA. Formaldehyde, formalin, paraformaldehyde and glutaraldehyde: What they are and what they do. Microsc today. 2000;1:8-12.

20.Lenardon MD, Munro CA, Gow NA. Chitin synthesis and fungal pathogenesis Curr Opin Microbiol. 2010 ;13(4):416-423. doi: 10.1016/j.mib.2010.05.002

21.Linder M, Winiecka-Krusnell J, Linder E. Use of recombinant cellulose-binding domains of Trichoderma reesei cellulase as a selective immunocytochemical marker for cellulose in protozoa. Appl Environ Microbiol. 2002;68(5):2503 2508.

22.Lorenzo-Morales J, Khan NA, Walochnik J. An update on Acanthamoeba keratitis: diagnosis, pathogenesis and treatment. Parasite. 2015;22:10. doi: $10.1051 /$ parasite/2015010

23.Lorenzo-Morales J, Martín-Navarro CM, Lopez-Arencibia A, Arnalich-Montiel F, Pinero JE, Valladares B. Acanthamoeba keratitis: an emerging disease gathering importance worldwide? Trends Parasitol. 2013;29(4):181-187. doi: 10.1016/j.pt.2013.01.006.

24.Marciano-Cabral F, Cabral G. Acanthamoeba spp. as agents of disease in humans. Clin Microbiol Rev. 2003;16(2):273-307.
25.Marines HM, Osato MS, Font RL. The value of calcofluor white in the diagnosis of mycotic and Acanthamoeba infections of the eye and ocular adnexa. Ophthalmol. 1987;94(1):23-26.

26.Martinez AJ, Visvesvara GS. Free-living, amphizoic and opportunistic amoebas. Brain Pathol. 1997;7(1):583-598.

27.Matuda T, Hamada M, Ota K. Pathogenic fungi in tissue and clinical material delineated by Fungiflora Y, a non-specific fluorescent staining. Jpn J Clin Dermatol. 1994;48:99-103.

28.Nagington J, Watson PG, Playfair TJ, McGill J, Jones BR, Steele AD. Amoebic infection of the eye. Lancet. 1974;304:1537-540.

29.Neff RJ, Benton WF, Neff RH. The composition of the mature cyst wall of soil amoeba Acanthamoeba sp. J Cell Biol. 1964;23:66A.

30.Pasricha G, Sharma S, Garg P, Aggarwal RK. Use of 18S rRNA gene-based PCR assay for diagnosis of Acanthamoeba keratitis in non-contact lens wearers in India. J Clin Microbiol. 2003;41:3206-3211.

31.Pöhlker C, Huffman JA, Pöschl U. Autofluorescence of atmospheric bioaerosols - fluorescent biomolecules and potential interferences. Atmos Meas Tech. 2011;4:5857-5933.

32.Radford CF, Bacon AS, Dart JK, Minassian DC. Risk factors for acanthamoeba keratitis in contact lens users: a case control study. BMJ. 1995;10:1567-1570.

33.Radford CF, Minassian DC, Dart JK. Acanthamoeba keratitis in England and Wales: incidence, outcome, and risk factors. Br J Ophthalmol. 2002;86(5):536542.

34.Schroeder JM, Booton GC, Hay J, Niszl IA, Seal DV, Markus MB. Use of subgenic 18S ribosomal DNA PCR and sequencing for genus and genotype identification of Acanthamoebae from humans with keratitis and from sewage sludge. J Clin Microbiol. 2001;39(5):1903-1911.

35.Schuster FL, Visvesvara GS. Amebic encephalitides and amebic keratitis caused by pathogenic and opportunistic free-living amebas. Curr Treat Options Infect Dis. 2003;5:273-282.

36.Sharma S, Garg P, Rao GN. Patient characteristics, diagnosis and treatment of non-contact lens related Acanthamoeba keratitis. Br J Ophthalmol. 2000;84(10):1103-1108.

37.Shirwadkar CG, Samant R, Sankhe M, Deshpande R, Yagi S, Schuster FL, Siram R, Visvesvara G. Acanthamoeba encephalitis in patient with systemic lupus, India. Emerg Infect Dis. 2006;12(6):984-986. doi: 10.3201/eid1206.060087

38.Siddiqui R, Khan NA. Biology and pathogenesis of Acanthamoeba. Parasite Vectors. 2012;5:6. doi: 10.1186/1756-3305-5-6

39.Singh P, Kochhar R, Vashishta RK, Khandelwal N, Prabhakar S, Mohindra S. Amoebic meningoencephalitis: spectrum of imaging findings. AJNR Am J Neuroradiol. 2006;27(6):1217-1221.

40.Spring KD, Davidson MW. Introduction to fluorescence microscopy.

41.Tomlinson $\mathrm{G}$, Jones $\mathrm{A}$. Isolation of cellulose from the cyst wall of a soil amoeba. Biochim Biophys Acta. 1962;63:194-200.

42.Visvesvara GS, Moura H, Schuster FL. Pathogenic and opportunistic freelivingamoebae: Acanthamoeba spp., Balamuthia mandrillaris, Naegleria fowleri, and Sappinia diploidea. FEMS Immunol Med Microbiol. 2007;50(1):126. doi: 10.1111/j.1574-695X.2007.00232.x

43.Visvesvara GS. Biology of Acanthamoeba and epidemiology of Acanthamoeba keratitis. Ophthalmol. 1991; 33:719-726.

44. Wagoner MD, Nemon SE. 1999. Concomitant Acanthamoeba and Streptococcus viridans keratitis. Middle East J Ophalmol.1999;2:152-156.

45.Walchik J, Oballer A, Aspok H. Correlations between molecular, physiological, andmorphologcal characteristics in clinical and nonclinical isolates of Acanthamoeba. Appl Environ Microbiol. 2000;66: 4408-4423. doi: 10.1128/ AEM.66.10.4408-4413.2000

46.Wilhelmus KR, Osato MS, Font RL, Robinson NM, Jones DB. Rapid diagnosis of Acanthamoeba Keratitis using Calcofluor White. Arch Ophthalmol. 1986;104(9):1309-1312.

47.Yang S, Wu X, Wang X, Samuelson LA, Cholli AL. Kumar J. Synthesis and Characterization of Fluorescent Cellulose. J Macromol Sci Pure Appl Chem. 2003;12:1275-1282. 